\title{
About the Error Term for Best Approximation with Respect to the Hausdorff Related Metrics*
}

\author{
K. Böröczky \\ Rényi Institute of Mathematics, Hungarian Academy of Sciences, \\ Pf. 127, H-1364 Budapest, Hungary \\ carlos@renyi.hu
}

\begin{abstract}
Let $M$ be a convex body with $C_{+}^{3}$ boundary in $\mathbb{R}^{d}, d \geq 3$, and consider a polytope $P_{n}$ (or $P_{(n)}$ ) with at most $n$ vertices (at most $n$ facets) minimizing the Hausdorff distance from $M$. It has long been known that as $n$ tends to infinity, there exist asymptotic formulae of order $n^{-2 /(d-1)}$ for the Hausdorff distances $\delta_{\mathrm{H}}\left(P_{n}, M\right)$ and $\delta_{\mathrm{H}}\left(P_{(n)}, M\right)$. In this paper a bound of order $n^{-5 /(2(d-1))}$ is given for the error of the asymptotic formulae.

This bound is clearly not the best possible, and Gruber [9] conjectured that if the boundary of $M$ is sufficiently smooth, then there exist asymptotic expansions for $\delta_{\mathrm{H}}\left(P_{n}, M\right)$ and $\delta_{\mathrm{H}}\left(P_{(n)}, M\right)$. With the help of quasiconformal mappings, we show for the three-dimensional unit ball that the error is at least $f(n) \cdot n^{-2}$ where $f(n)$ tends to infinity. Therefore in this case, no asymptotic expansion exists in terms of $n^{-2 /(d-1)}=n^{-1}$.
\end{abstract}

\section{Introduction}

Assume that $M$ is a convex body with $C_{+}^{2}$ boundary in $\mathbb{R}^{d}$, and $\delta$ is one of the notions of distance among the Hausdorff metric, the Banach-Mazur metric, or Schneider's notion of distance. Consider a polytope $P_{n}$ (or $P_{(n)}$ ) with at most $n$ vertices (at most $n$ facets) minimizing $\delta\left(M, P_{n}\right)$ (or $\delta\left(M, P_{(n)}\right)$ ). In the last two decades, much effort and many brilliant ideas have been put into obtaining asymptotic formulae for $\delta\left(M, P_{n}\right)$ and $\delta\left(M, P_{(n)}\right)$ as $n$ tends to infinity. After initial ideas by $L$. Fejes Tóth (in dimensions 2 and 3) [6] and results by McClure and Vitale in the plane [13], it was Schneider who provided for the first time an asymptotic formula in higher dimensions [14]; namely, he considered the Hausdorff distance for convex bodies with $C_{+}^{3}$ boundary. A new understanding of the problem came by the method developed by Gruber [7]. This method could handle all

\footnotetext{
* This research was supported by OTKA 031984, Hungary.
} 
related questions (say for the Banach-Mazur distance), and the the assumption on the boundary could be relaxed to $C_{+}^{2}$. See Gruber's comprehensive surveys [8] and [9] for a detailed history of this field. The central problem of this paper is to estimate the error of these asymptotic formulae as $n$ tends to infinity.

First we introduce the notions of distance of convex bodies $K$ and $P$ which we consider. Note that the support function of a convex body $K$ is defined as $h_{K}(u)=$ $\max _{x \in K}\langle x, u\rangle$.

Hausdorff metric: $\delta_{\mathrm{H}}(K, P)=\max _{u \in S^{d-1}}\left|h_{\mathrm{M}}(u)-h_{P}(u)\right|$; namely, the maximum of the distances of the points of $K$ from $P$, and the distances of the points of $P$ from $K$.

Banach-Mazur metric: Let the interiors of $K$ and $P$ contain the origin. Then $\delta_{\mathrm{BM}}(K, P)$ is the minimum of $\ln \lambda$ such that there exists a linear transformation $T$ satisfying $T P \subset K \subset \lambda \cdot T P$.

Schneider's distance: Let $P$ be contained in $K$. Then $\delta_{\mathrm{SCH}}(K, P)$ is the maximal volume of a cap cut off from $K$ by a supporting hyperplane of $P$.

Observe that the Hausdorff metric is a metric on the space of convex bodies, while the Banah-Mazur distance is a metric for convex bodies which contain the origin in their interiors if convex bodies and their linear images are identified. On the other hand, Schneider's notion of distance is not a metric, and actually not so important as the other two notions.

We consider a convex body $M$. Then $\partial M$ can be covered by a family of open neighborhoods on $\partial M$ such that each of them is the graph of some convex function in $d-1$ variables. If each function is $C^{k}, k \geq 2$, then $\partial M$ is a $C^{k}$ manifold. In this case, the second fundamental form $Q_{x}$ can be defined at each $x \in \partial M$, and the Gauss curvature $\kappa(x)$ is the determinant of $Q_{x}$. The convexity of $M$ yields that $Q_{x}$ is positive semidefinite, and we say that $M$ is $C_{+}^{k}$ if the second fundamental form $Q_{x}$ is positive definite at each $x \in \partial M$; or, equivalently, if $\kappa(x)$ is positive at each $x$. The basic reference about the properties of smooth convex bodies is the book [15] by Schneider, and see Section 2.2 for the properties we need.

Next we list the known asymptotic formulae where $P_{n}\left(P_{(n)}\right)$ denotes a best approximating polytope with at most $n$ vertices (facets) with respect to the given metric. If $\partial M$ is $C_{+}^{2}$ then these results were proved by Gruber (see [8] and [9] for references). Integration is with respect to the induced measure on $\partial M$ as a $C^{2}$ hypersurface in $\mathbb{R}^{d}$, which measure coincides with the Hausdorff $(d-1)$-measure. The exterior unit normal at a point $x \in \partial M$ is denoted by $\nu(x)$. Finally, we denote the minimal density of a covering of $\mathbb{R}^{d-1}$ with congruent balls by $\vartheta_{d-1}$, and the volume of the $(d-1)$-dimensional unit ball by $\kappa_{d-1}$. Then

$$
\begin{aligned}
\delta_{\mathrm{H}}\left(M, P_{n}\right), \delta_{\mathrm{H}}\left(M, P_{(n)}\right) \sim & \frac{1}{4}\left(\frac{\vartheta_{d-1}}{\kappa_{d-1}}\right)^{2 /(d-1)}\left(\int_{\partial M} \kappa(x)^{1 / 2} d x\right)^{2 /(d-1)} \cdot \frac{1}{n^{2 /(d-1)}} \\
\delta_{\mathrm{BM}}\left(M, P_{n}\right), \delta_{\mathrm{BM}}\left(M, P_{(n)}\right) \sim & \frac{1}{2}\left(\frac{\vartheta_{d-1}}{\kappa_{d-1}}\right)^{2 /(d-1)}\left(\int_{\partial M} \frac{\kappa(x)^{1 / 2}}{h_{M}(v(x))^{(d-1) / 2}} d x\right)^{2 /(d-1)} \\
& \frac{1}{n^{2 /(d-1)}}
\end{aligned}
$$




$$
\begin{aligned}
\delta_{\mathrm{CH}}\left(M, P_{n}\right), \delta_{\mathrm{SCH}}\left(M, P_{(n)}\right) \sim & \frac{\kappa_{d-1}}{d+1} \cdot\left(\frac{\vartheta_{d-1}}{\kappa_{d-1}}\right)^{(d+1) /(d-1)} \\
& \cdot\left(\int_{\partial M} \kappa(x)^{1 /(d+1)} d x\right)^{(d+1) /(d-1)} \frac{1}{n^{(d+1) /(d-1)}} .
\end{aligned}
$$

In the case of the Hausdorff metric, the same asymptotic formulae are proved assuming that $P_{n}$ is inscribed, or that $P_{n}$ is circumscribed (only with double constant). The integral which appears in the formula for the Banach-Mazur distance is the so-called centro affine surface area, and it is invariant under linear transformations (see [3] or [11]). In addition, the integral which appears in the formula for Schneider's distance is the so-called affine surface area, and it is invariant under volume-preserving affine transformations (see [3] or [11]). The goal of this paper is to estimate the error term for the formulae. We write $f=O(g)$ if $|f| \leq c \cdot g$ where $c$ depends only on $M$.

Theorem 1.1. Let $M$ be a convex body with $C_{+}^{3}$ boundary, and assume that $P_{n}$ is some polytope with at most $n$ vertices.

(i) If $P_{n}$ minimizes $\delta_{\mathrm{H}}\left(M, P_{n}\right)$, then

$$
\begin{aligned}
\delta_{\mathrm{H}}\left(M, P_{n}\right)= & \frac{1}{4}\left(\frac{\vartheta_{d-1}}{\kappa_{d-1}}\right)^{2 /(d-1)}\left(\int_{\partial M} \kappa(x)^{1 / 2} d x\right)^{2 /(d-1)} \\
& \cdot \frac{1}{n^{2 /(d-1)}}+O\left(\frac{1}{n^{5 / 2(d-1)}}\right)
\end{aligned}
$$

If $P_{n}$ is inscribed or circumscribed, then $\delta_{\mathrm{H}}\left(M, P_{n}\right)$ is twice the value above.

(ii) If $o \in$ int $M$, int $P_{n}$ and $P_{n}$ minimizes $\delta_{\mathrm{BM}}\left(M, P_{n}\right)$, then

$$
\begin{aligned}
\delta_{\mathrm{BM}}\left(M, P_{n}\right)= & \frac{1}{2}\left(\frac{\vartheta_{d-1}}{\kappa_{d-1}}\right)^{2 /(d-1)}\left(\int_{\partial M} \frac{\kappa(x)^{1 / 2}}{h(\nu(x))^{(d-1) / 2}} d x\right)^{2 /(d-1)} \\
& \cdot \frac{1}{n^{2 /(d-1)}}+O\left(\frac{1}{n^{5 / 2(d-1)}}\right)
\end{aligned}
$$

(iii) If $P_{n} \subset M$ and $P_{n}$ minimizes $\delta_{\mathrm{SCH}}\left(M, P_{n}\right)$, then

$$
\begin{aligned}
\delta_{\mathrm{SCH}}\left(M, P_{n}\right)= & \frac{\kappa_{d-1}}{d+1}\left(\frac{\vartheta_{d-1}}{\kappa_{d-1}}\right)^{(d+1) /(d-1)}\left(\int_{\partial M} \kappa(x)^{1 /(d+1)} d x\right)^{(d+1) /(d-1)} \\
& \cdot \frac{1}{n^{(d+1) /(d-1)}}+O\left(\frac{1}{n^{(2 d+3) / 2(d-1)}}\right)
\end{aligned}
$$

The same formulae hold if $P_{n}$ is replaced by some polytope $P_{(n)}$ with at most $n$ facets.

It is clear from the proof of Theorem 1.1 that Theorem 1.1 holds even if the only assumption on the boundary of $M$ is that it is $C_{+}^{2}$, and the coefficients of the second fundamental form $Q_{x}$ are Lipschitz functions of the local parameters.

We also show (see Corollary 3.1) that the analogous formulae hold for convex hypersurfaces which are open subsets of the boundary of some convex body, only one needs the appropriate definition of approximating polytopal hypersurfaces. 
Next we discuss possible improvements on the error term. For a convex domain $M$ in $\mathbb{R}^{2}$, let $P_{n}$ be some convex polygon with at most $n$ vertices containing $M$. If the boundary of $M$ is $C_{+}^{4}$ and $P_{n}$ minimizes the Hausdorff distance from $M$, then

$$
\delta_{\mathrm{H}}\left(M, P_{n}\right)=a_{2}(M) \cdot \frac{1}{n^{2}}+a_{4}(M) \cdot \frac{1}{n^{4}}+o\left(\frac{1}{n^{4}}\right)
$$

holds (see [12]) where $a_{2}(M)$ is given above, and $a_{4}(M)$ depends on $M$. We remark that if the boundary of $M$ is $C_{+}^{\infty}$ and the area of $P_{n}$ is maximal, then there exists an infinity expansion for the area of $M \backslash P_{n}$ in terms of $1 / n^{2}$ (see [16]). These facts point to the conjecture of [9] that for sufficiently smooth convex bodies with positive curvature in $\mathbb{R}^{d}$, there exists an asymptotic expansion of $\delta\left(M, P_{n}\right)$.

In order to obtain more information about the expected order of the error term in higher dimensions, assume that $M$ is the $d$-dimensional unit ball $B^{d}, d \geq 3$. Let $P_{n}\left(P_{(n)}\right)$ be the polytope with at most $n$ vertices (facets) which minimizes $\delta_{\mathrm{H}}\left(B^{\overline{2}}, P_{n}\right)\left(\delta_{\mathrm{H}}\left(B^{2}, P_{(n)}\right)\right)$. We prove that

$$
\delta_{\mathrm{H}}\left(B^{d}, P_{n}\right)=\frac{1}{4}\left(\frac{d \kappa_{d} \vartheta_{d-1}}{\kappa_{d-1}}\right)^{2 /(d-1)} \cdot \frac{1}{n^{2 /(d-1)}}+O\left(\frac{1}{n^{8 / 3(d-1)}}\right) .
$$

We note that the analogous estimates follow directly for the Banach-Mazur distance or Schneider's notion of distance. If $d=3$, then an equivalent estimate was proved in the Masters thesis [5] of G. Fejes Tóth utilizing the method of van der Waerden [17].

As a lower bound on the error term, we prove that

$$
\delta_{\mathrm{H}}\left(B^{3}, P_{n}\right)>\frac{2 \pi}{3 \sqrt{3}} \cdot \frac{1}{n}+\frac{f(n)}{n^{2}},
$$

where $f(n)$ tends to infinity. This shows that $\delta_{H}\left(B^{3}, P_{n}\right)$ cannot have a series expansion in terms of $n^{-2 /(d-1)}=1 / n$. It seems to be plausible that the right order of the error term in Theorem 1.1 is $n^{-3 /(d-1)}$ if $d \geq 3$. Exactly the same can be said about the error term if the polytopes are assumed to be inscribed or circumscribed.

Remark about Packing and Covering. Approximation of $B^{d}$ by polytopes in the Hausdorff sense is intimately connected to the problem of covering $S^{d-1}$ with equal balls. Let $R_{n}\left(S^{d-1}\right)\left(r_{n}\left(S^{d-1}\right)\right)$ be the minimal (maximal) radius of $n$ equal balls in $S^{d-1}$ which form a covering (packing). Denote the maximal density of packing equal balls into $\mathbb{R}^{d-1}$ by $\delta_{d-1}$. The method of proving the results above yields the following statements:

$$
\begin{aligned}
& R_{n}\left(S^{d-1}\right)=\left(\frac{d \kappa_{d} \vartheta_{d-1}}{\kappa_{d-1}}\right)^{1 /(d-1)} \cdot \frac{1}{n^{1 /(d-1)}}+O\left(\frac{1}{n^{5 / 3(d-1)}}\right), \\
& r_{n}\left(S^{d-1}\right)=\left(\frac{d \kappa_{d} \delta_{d-1}}{\kappa_{d-1}}\right)^{1 /(d-1)} \cdot \frac{1}{n^{1 /(d-1)}}+O\left(\frac{1}{n^{5 / 3(d-1)}}\right) .
\end{aligned}
$$

On the other hand, we prove that

$$
R_{n}\left(S^{2}\right)>\sqrt{\frac{8 \pi}{3 \sqrt{3}}} \cdot \frac{1}{\sqrt{n}}+\frac{f_{0}(n)}{n^{3 / 2}} \text { and } r_{n}\left(S^{2}\right)<\sqrt{\frac{2 \pi}{\sqrt{3}}} \cdot \frac{1}{\sqrt{n}}-\frac{f_{0}(n)}{n^{3 / 2}},
$$

where $f_{0}(n)$ tends to infinity. While the upper estimates for the error terms have been 
known if $d=3$ (see [17] and [5]), the trivial lower estimate of order $n^{-\frac{3}{2}}$ had not been improved before in spite of various efforts.

The paper is organized as follows: First we prove Theorem 1.1, then we extend it to Jordan measurable subsets of the boundary of $\partial M$. Finally, we verify the lower bound on the error term.

\section{The Proof of Theorem 1.1}

We start with coverings by congruent ellipsoids in $\mathbb{R}^{d-1}$, what is followed by constructing almost flat patches on $\partial M$. We verify Theorem 1.1 first for the Hausdorff metric and for $P_{n}$ in order to present the main ideas, then we consider the other metrics, and we close the section by explaining the necessary alterations for $P_{(n)}$.

\subsection{Coverings in $\mathbb{R}^{d-1}$}

We consider coverings of a Jordan measurable set $J$ in $\mathbb{R}^{d-1}$. For $r>0$, the Minkowski sum $J+r B^{d-1}$ is the parallel domain with radius $r$, and if $J$ contains some ball of radius $r$, then let $J \ominus r B^{d-1}$ be the family of the centers of all $r$-dimensional balls which are contained in $J$. The Hausdorff $(d-1)$-measure is denoted by $|\cdot|$.

Lemma 2.1. Assume that a closed Jordan measurable set $J$ in $\mathbb{R}^{d-1}$ contains some ball of radius $r>0$, and denote the minimal number of balls with radius $r$ which cover $J$ by $N_{J}(r)$. Then

$$
\frac{\vartheta_{d-1}}{\kappa_{d-1}} \cdot \frac{\left|J \ominus r B^{d-1}\right|}{r^{d-1}} \leq N_{J}(r) \leq \frac{\vartheta_{d-1}}{\kappa_{d-1}} \cdot \frac{\left|J+r B^{d-1}\right|}{r^{d-1}} .
$$

Proof. For $a>0$, denote by $\mathcal{T}_{a}^{d-1}$ the torus $\mathbb{R}^{d-1} / a \mathbb{Z}^{d-1}$, equipped with the Haar measure induced by the Lebesgue measure in $\mathbb{R}^{d-1}$. If $J^{\prime}$ is a Jordan measurable subset and $\Gamma$ is a finite subset of $\mathcal{T}_{a}^{d-1}$, then

$$
\int_{\mathcal{T}_{a}^{d-1}} \#\left(\left(x+J^{\prime}\right) \cap \Gamma\right) d x=\left|J^{\prime}\right| \cdot \# \Gamma .
$$

For $a>2 r$, let $N_{a}(r)$ be the minimal number of $r$ balls in $\mathcal{T}_{a}^{d-1}$ which cover $\mathcal{T}_{a}^{d-1}$, and denote by $\Gamma_{a}(r)$ a set of centers of $N_{a}(r)$ balls with radius $r$ in $\mathcal{T}_{a}^{d-1}$ which cover $\mathcal{T}_{a}^{d-1}$. We use the following property of $\vartheta_{d-1}$ : for any $\varepsilon>0$, there exists a positive $a_{0}(\varepsilon)$ such that if $a>a_{0}(\varepsilon) \cdot r$, then

$$
(1-\varepsilon) \cdot \frac{\vartheta_{d-1}}{\kappa_{d-1}} \cdot \frac{a^{d-1}}{r^{d-1}} \leq N_{a}(r) \leq(1+\varepsilon) \cdot \frac{\vartheta_{d-1}}{\kappa_{d-1}} \cdot \frac{a^{d-1}}{r^{d-1}} .
$$

In the argument below, after choosing $\varepsilon$, we always fix some $a>a_{0}(\varepsilon) \cdot r$ with $a>$ $\operatorname{diam} J+4 r$, and assume that $J+r B^{d-1}$ is embedded into $\mathcal{T}_{a}^{d-1}$.

Let $\varepsilon>0$. Now (6) yields that there exists a copy of $J$ which is covered by at most $a^{-(d-1)} \cdot\left|J+r B^{d-1}\right| \cdot N_{a}(r)$ balls of radius $r$, and the upper bound for $N_{J}(r)$ is a consequence of (7) and of the arbitrariness of $\varepsilon$. 
Next, we suppose indirectly that

$$
N_{J}(r)<(1-\varepsilon) \cdot \frac{\vartheta_{d-1}}{\kappa_{d-1}} \cdot \frac{\left|J \ominus r B^{d-1}\right|}{r^{d-1}}
$$

for some positive $\varepsilon$. Now (6) and (7) yield that there exists a copy of $x+J \ominus r B^{d-1}$ which contains at least

$$
a^{-(d-1)} \cdot\left|J \ominus r B^{d-1}\right| \cdot N_{a}(r) \geq(1-\varepsilon) \cdot \frac{\vartheta_{d-1}}{\kappa_{d-1}} \cdot \frac{\left|J \ominus r B^{d-1}\right|}{r^{d-1}}
$$

centers. Replace the balls whose center is in $x+J \ominus r B^{d-1}$ with $N_{J}(r)$ balls with radius $r$ which cover $x+J$. This way we obtain a new family of balls covering $\mathcal{T}_{a}^{d-1}$, and the indirect assumption yields that the cardinality of the new family is less than $N_{a}(r)$. This contradiction finally proves the lemma.

Lemma 2.1 was proved in [2] using a more complicated argument.

Let $q$ be a positive definite quadratic form in $\mathbb{R}^{d-1}$, and we denote the set of points satisfying $q(x) \leq 1$ by $B_{q}$. If $C$ is a convex body, then we denote the inradius with respect to $q$ by $\varrho_{q}(C)$; namely, $\varrho_{q}(C)$ is the maximum of $Q$ such that a translate of $Q B_{q}$ is contained in $C$. In addition, $N_{C, q}(r)$ denotes the minimal number of translates of $r \cdot B_{q}$ which cover $C$.

The inradius with respect to the Euclidean metric is denoted by $Q(C)$. If $r<Q(C)$, then readily

$$
\left(1-\frac{r}{\varrho(C)}\right)^{d-1} \cdot|C| \leq\left|C \ominus r B^{d-1}\right|<\left|C+r B^{d-1}\right| \leq\left(1+\frac{r}{\varrho(C)}\right)^{d-1} \cdot|C| .
$$

Therefore transforming $q$ into the quadratic form $q_{0}(u)=\langle u, u\rangle$ and Lemma 2.1 yield

Corollary 2.2. Let $C$ be a convex body and let $q$ be a positive definite quadratic form in $\mathbb{R}^{d-1}$. If $r<\frac{1}{2} \cdot Q_{q}(C)$, then

$$
N_{C, q}(r)=\left(1+O\left(\frac{r}{\varrho_{q}(C)}\right)\right) \cdot \frac{\vartheta_{d-1}}{\kappa_{d-1}} \cdot \frac{\sqrt{\operatorname{det} q} \cdot|C|}{r^{d-1}} .
$$

\subsection{Subdividing the Boundary}

Let $M$ be a convex body with $C^{2}$ boundary. For some $x \in \partial M$, denote the tangent hyperplane at $x$ by $H_{x}$, and hence an open neighborhood $U$ of $x$ in $\partial M$ is the graph of a convex $C^{2}$ function $f$ defined in the projection $V_{x}$ of $U$ into $H_{x}$. Denote the derivative of $f$ at $z$ by $l_{z}$ and the quadratic form representing the second derivative of $f$ by $q_{z}$. We deduce using the Taylor expansion of $f$ that if $y, z \in V_{x}$, then

$$
f(z)=f(y)+l_{y}(z-y)+\frac{1}{2} q_{w}(z-y),
$$

where $w=y+t(z-y)$ for some $0<t<1$. In particular, the second fundamental form at $x$ is $Q_{x}=q_{x}$. 
Now let $\partial M$ be $C_{+}^{2}$, and assume that the coefficients of the second fundamental form are Lipschitz functions of the local parameters. Then $q_{z}$ is positive definite for $z \in V_{x}$, and $q_{z}$ is a Lipschitz function of $z$. Equip the tangent space at $x \in \partial M$ with the second fundamental form $Q_{x}$, and consider $\partial M$ as a Riemannian manifold with this metric. For $x, y \in \partial M$, we denote the distance of $x$ and $y$ in this metric by $d_{\partial M}(x, y)$. In addition, we denote the Euclidean distance by $d(\cdot, \cdot)$. We note that there exists a $d_{0}>0$ such that $x, y \in \partial M$ and $d(x, y)<d_{0}$ yield that

$$
d_{\partial M}(x, y) \approx d(x, y) \text { and } \angle(v(x), v(y)) \approx d(x, y) .
$$

For small $\omega>0$, fix a maximal system $\left\{x_{1}, \ldots, x_{k_{\omega}}\right\}$ of points on $\partial M$ such that $d\left(x_{i}, x_{j}\right) \geq \omega$. Then for any $x \in \partial M$ there exists an $x_{i}$ satisfying $d\left(x, x_{i}\right)<2 \omega$. We define $P_{\omega}$ as the polytope bounded by the tangent hyperplanes $H_{x_{i}}, i=1, \ldots, k_{\omega}$, and we denote the facet of $P_{\omega}$ touching at $x_{i}$ by $F_{i}$, and the image of $F_{i}$ and $2\left(F_{i}-x_{i}\right)+x_{i}$ by the closest point map into $\partial M$ by $\Omega_{i}$ and by $\widetilde{\Omega}_{i}$, respectively.

Lemma 2.3. Let $M$ be a convex body with $C_{+}^{2}$ boundary, and assume that the coefficients of $Q_{x}$ are Lipschitz functions of the local parameters on $\partial M$. Let $\omega>0$ be small, and hence $k_{\omega} \approx \omega^{-(d-1)}$. In addition, fix an $i \leq k_{\omega}$, and denote the orthogonal projection from $\widetilde{\Omega}_{i}$ into aff $F_{i}$ by'. Then

(i) $\varrho\left(F_{i}\right)>\frac{1}{3} \omega$ and $\left|F_{i}\right| \approx \omega^{d-1}$;

(ii) $\left(1-O\left(\omega^{2}\right)\right) \cdot\left(F_{i}-x_{i}\right)+x_{i} \subset \Omega_{i}^{\prime} \subset F_{i}$;

(iii) if $x, y \in \widetilde{\Omega}_{i}$, then

$$
\begin{aligned}
& d(x, y)=\left(1+O\left(\omega^{2}\right)\right) \cdot d\left(x^{\prime}, y^{\prime}\right) \quad \text { and } \\
& d_{\partial M}(x, y)=(1+O(\omega)) \cdot \sqrt{Q_{x_{i}}\left(x^{\prime}-y^{\prime}\right)}
\end{aligned}
$$

(iv) $\left|\Omega_{i}\right|=\left(1+O\left(\omega^{2}\right)\right) \cdot\left|F_{i}\right|$ and

$$
\int_{\Omega_{i}} \sqrt{\kappa(x)} d x=(1+O(\omega)) \cdot \sqrt{\operatorname{det} Q_{x_{i}}} \cdot\left|F_{i}\right|
$$

Proof. Since $\angle\left(v(x), v\left(x_{i}\right)\right)=O(\omega)$ holds for $x \in \widetilde{\Omega}_{i}$ by (9), we deduce that the inradius of $F_{i}$ is at least $\frac{1}{3} \omega$, and the circumradius is at most $3 \omega$ for small $\omega$. This yields $k_{\omega} \approx \omega^{-(d-1)}$ and (i). In addition, (ii) follows as $d\left(x, x^{\prime}\right)=O\left(\omega^{2}\right)$ holds for $x \in \widetilde{\Omega}_{i}$. Finally, (9) yields that $\left\langle\nu(x), \nu\left(x_{i}\right)\right\rangle=1-O\left(\omega^{2}\right)$ holds for $x \in \widetilde{\Omega}_{i}$, and hence (iii) and (iv) are consequences of (8), and the fact that the second fundamental form is Lipschitz.

\subsection{Polytopal Approximation}

Let $M$ be a smooth convex body, let $P$ be a polytope in $\mathbb{R}^{d}$, and assume that $x \in \partial M$ and $y \in \partial P$ satisfy that $d(x, y)=\delta_{\mathrm{H}}(M, P)$. We use, without mentioning, the following:

Fact. $y-x$ is a normal to $\partial M$ at $x$. If $y \notin M$, then $y$ is a vertex of $P$. If $y \in M$, then $y$ lies in the relative interior of a facet of $P$ and $y-x$ is a normal to this facet. 
First we consider approximation of the unit ball $B^{d}$ because this case is much simpler.

Lemma 2.4. If $P_{n}$ is a polytope with at most $n$ vertices which minimizes the Hausdorff distance from $B^{d}$, then

$$
\delta_{\mathrm{H}}\left(P_{n}, B^{d}\right)=\left(1+O\left(n^{-2 /(d-1)}\right)\right) \cdot \frac{1}{4} \cdot R_{n}\left(S^{d-1}\right)^{2} .
$$

If $P_{n}$ is assumed to be inscribed or circumscibed, then $\frac{1}{4}$ should be replaced by $\frac{1}{2}$.

Proof. We observe that if the spherical distance of $x, y \in S^{d-1}$ is $R$, then

$$
\begin{aligned}
\langle v(x), v(y)\rangle & =1-O\left(R^{2}\right) \\
\langle x-y, v(x)\rangle & =\left(1+O\left(R^{2}\right)\right) \cdot \frac{1}{2} R^{2} .
\end{aligned}
$$

In addition, we have $R_{n}\left(S^{d-1}\right) \approx n^{-1 /(d-1)}$, and let $\Sigma_{n}$ be a family of centers in a covering of $S^{d-1}$ by $n$ spherical balls of radius $R_{n}\left(S^{d-1}\right)$.

First assume that $P_{n} \subset B^{d}$, and hence the vertex set is contained in $S^{d-1}$. The affine hull of each facet of $P_{n}$ cuts off a spherical ball from $S^{d-1}$. Since the maximal radius of these balls equals the minimal radius of spherical balls centered at the vertices which cover $S^{d-1}$, we deduce

$$
\delta_{\mathrm{H}}\left(P_{n}, B^{d}\right) \geq \frac{1}{2} \cdot R_{n}\left(S^{d-1}\right)^{2} \cdot\left(1+O\left(n^{-2 /(d-1)}\right)\right)
$$

by (10). The convex hull of $\Sigma_{n}$ yields an upper bound of the same order for $\delta_{\mathrm{H}}\left(P_{n}, B^{d}\right)$.

Next, assume that $P_{n}$ is circumscribed around $B^{d}$. Let $\Sigma \subset S^{d-1}$ be the set of radial projections of the vertices of $P_{n}$, and hence there exists an $x \in \Sigma$ such that the spherical distance of $x$ from any points of $\Sigma$ is at least $R_{n}\left(S^{d-1}\right)$. Now there exist vertices $v_{1}, \ldots, v_{d}$ of $P_{n}$ such that their convex hull lies on $\partial P_{n}$, and the radial projection of the convex hull contains $x$. Denote by $x_{i}$ the radial projection of $v_{i}$ into $S^{d-1}$. Since aff $\left\{v_{1}, \ldots, v_{d}\right\}$ avoids int $B^{d}$, we may assume that $\left\langle x-v_{1}, v(x)\right\rangle \leq 0$, and hence (10) yields (11). In order to construct a well approximating polytope, move each point of $\Sigma_{n}$ outwards radially with a distance of

$$
\frac{1}{2} \cdot R_{n}\left(S^{d-1}\right)^{2} \cdot\left(1+c \cdot n^{-2 /(d-1)}\right)
$$

for a suitable positive constant $c$, and let $Q_{n}$ be the convex hull of these points. If $c$ is chosen large enough, then (10) yields that $Q_{n}$ contains $B^{d}$, and also yields the required upper bound for $\delta_{\mathrm{H}}\left(Q_{n}, B^{d}\right)$.

Finally, in case of general approximation, construct $\Sigma, x$, and $v_{1}, \ldots, v_{d}$ as above. If there exists a $v_{i}$ with

$$
\left\langle x-v_{i}, v(x)\right\rangle \leq \frac{1}{4} \cdot R_{n}\left(S^{d-1}\right)^{2},
$$

then the distance of this $v_{i}$ from $B^{d}$ is suitably large, and the distance of aff $\left\{v_{1}, \ldots, v_{d}\right\}$ and $x$ is suitably large otherwise. For an upper bound on $\delta_{\mathrm{H}}\left(P_{n}, B^{d}\right)$, move each point of $\Sigma_{n}$ outwards radially with a distance of $\frac{1}{4} \cdot R_{n}\left(S^{d-1}\right)^{2}$, and take the convex hull. 
For $r<1$, denote by $N_{S^{d-1}}(r)$ the minimal number of spherical balls of radius $r$ which cover $S^{d-1}$. We deduce by Lemma 2.4 that (1) is equivalent to the estimate

$$
N_{S^{d-1}}(r)=\left(1+O\left(r^{2 / 3}\right)\right) \cdot \frac{d \kappa_{d} \vartheta_{d-1}}{\kappa_{d-1}} \cdot \frac{1}{r^{d-1}} .
$$

We apply Lemma 2.3 with $\omega=r^{1 / 3}$ and $M=B^{d}$, and we consider the $P_{\omega}$ given by Lemma 2.3. Denote the unit $(d-1)$-ball centered at the origin and parallel to $F_{i}$ by $B_{i}$.

Assume that $S^{d-1}$ is covered by $N_{S^{d-1}}(r)$ spherical balls of radius $r$. Project the $n_{i}$ balls which are completely contained in $\Omega_{i}$ orthogonally into $F_{i}$. Then the projection of each ball can be covered by a $(d-1)$-ball of radius $\left(1+O\left(r^{2 / 3}\right)\right) \cdot r$ by Lemma 2.3(iii), and the projections cover $F_{i} \ominus c \cdot r B_{i}$ for a $c$ depending only on $d$ by Lemma 2.3(ii). We deduce by Corollary 2.2 and Lemma 2.3(i) that

$$
n_{i}=\left(1+O\left(r^{2 / 3}\right)\right) \cdot \frac{\vartheta_{d-1}}{\kappa_{d-1}} \cdot \frac{\left|F_{i}\right|}{r^{d-1}} .
$$

On the other hand, readily $\sum n_{i} \leq N_{S^{d-1}}(r)$, and hence Lemma 2.3 (iv) yields that

$$
N_{S^{d-1}}(r) \geq\left(1+O\left(r^{2 / 3}\right)\right) \cdot \frac{\vartheta_{d-1}}{\kappa_{d-1}} \cdot \frac{d \kappa_{d}}{r^{d-1}}
$$

In order to obtain an upper bound on $N_{S^{d-1}}(r)$, we may choose a $c$ depending on $d$ with the help of Lemma 2.3(iii) such that if the spherical distance of $x, y \in \widetilde{\Omega}_{i}$ is $r$, then $d\left(x^{\prime}, y^{\prime}\right)>\left(1-c \cdot r^{2 / 3}\right) \cdot r$. We cover each $F_{i}$ by the minimal number $n_{i}$ of balls of radius $\left(1-c \cdot r^{2 / 3}\right) \cdot r$. Then projecting the centers orthogonally to aff $F_{i}$ into $\widetilde{\Omega}_{i}$, we obtain a covering of $\Omega_{i}$ by $n_{i}$ spherical balls of radius $r$. Here the same formula holds for $n_{i}$ as above, therefore we conclude (12), and in turn (1).

Next, let $M$ be a convex body with $C_{+}^{2}$ boundary, and assume that the coefficients of $Q_{x}$ are Lipschitz functions of the local parameters on $\partial M$. We denote by $R_{n}(\partial M)$ the minimal $R$ such that on $\partial M$, some $n$ balls of radius $R$ with respect to the metric determined by the second fundamental form cover $\partial M$.

Lemma 2.5. If $P_{n}$ is a polytope with at most $n$ vertices which minimizes the Hausdorff distance from $M$, then

$$
\delta_{\mathrm{H}}\left(P_{n}, M\right)=\left(1+O\left(n^{-1 /(d-1)}\right)\right) \cdot \frac{1}{4} \cdot R_{n}(\partial M)^{2} .
$$

If $P_{n}$ is assumed to be inscribed or circumscribed, then $\frac{1}{4}$ should be replaced by $\frac{1}{2}$.

Proof. We deduce by (8) that if $d_{\partial M}(x, y)=R$ is small, then

$$
\begin{aligned}
\langle v(x), v(y)\rangle & =1-O\left(R^{2}\right), \\
\langle x-y, v(x)\rangle & =(1+O(R)) \cdot \frac{1}{2} R^{2} .
\end{aligned}
$$

Since $R_{n}(\partial M) \approx n^{-l /(d-1)}$, the proof can be completed analogously to the proof of Lemma 2.4 . 
For small $r$, denote by $N_{\partial M}(r)$ the minimal number of balls of radius $r$ with respect to the metric determined by the second fundamental form which cover $\partial M$. We deduce by Lemma 2.5 that Theorem 1.1 for $P_{n}$ and for the Hausdorff metric is equivalent to the estimate

$$
N_{\partial M}(r)=\left(1+O\left(r^{1 / 2}\right)\right) \cdot \frac{\vartheta_{d-1}}{\kappa_{d-1}} \cdot \int_{\partial M} \kappa(x)^{1 / 2} d x \cdot \frac{1}{r^{d-1}} .
$$

For both the upper bound and the lower bound on $N_{\partial M}(r)$, apply Lemma 2.3 with $\omega=r^{1 / 2}$. Then (14) can be verified analogously to (12).

\subsection{The Banach-Mazur Metric and Schneider's Distance}

These notions of distance can be dealt with in a similar way as was done for the Hausdorff metric. If $M=B^{d}$, then the formulae follow directly from the estimates for the Hausdorff metric. So let $\partial M$ be $C_{+}^{2}$, and assume that the coefficients of the second fundamental form are Lipschitz functions of the local parameters.

We consider the Banach-Mazur distance. First we write it in a form which is more suitable in our context: Let $P$ be some polytope containing the origin in its interior. Assume that $\left\{F_{j}\right\}$ is the family of facets of $P$, and $x_{j} \in \partial M$ is the point where the exterior normals coincide with the exterior normals to $F_{j}$. Then $M \subset P$ is equivalent to saying that for each $j$, we have $h_{M}\left(v\left(x_{j}\right)\right) \leq h_{P}\left(v\left(x_{j}\right)\right)$. Now assume that $P \subset M$ and $s_{j}$ is the distance of $x_{j}$ from aff $F_{j}$ (the depth of the corresponding cap). Therefore the minimal $\lambda$ satisfying $M \subset \lambda \cdot P$ is

$$
\lambda=1+\max _{j}\left(1-\frac{s_{j}}{h_{M}\left(\nu\left(x_{j}\right)\right)}\right)^{-1} \cdot \frac{s_{j}}{h_{M}\left(v\left(x_{j}\right)\right)} .
$$

For $x \in \partial M$, consider the quadratic form $\widetilde{Q}_{x}=1 / h_{M}(v(x)) \cdot Q_{x}$ on the tangent hyperplane, whose coefficients are Lipschitz functions of the local parameters. We deduce by (15) that the same argument as for the Hausdorff metric gives the result for the Banach-Mazur distance, only the Riemannian metric on $\partial M$ determined by $Q_{x}$ should be replaced by the Riemannian metric determined by $\widetilde{Q}_{x}$.

We turn to Schneider's notion of distance. Assume that $P_{n} \subset M$ is a polytope with at most $n$ vertices such that $\delta_{\mathrm{SCH}}\left(M, P_{n}\right)$ is minimal. It follows that for large $n$, the cap avoiding int $P_{n}$ whose volume is $\delta_{\mathrm{SCH}}\left(M, P_{n}\right)$ is cut off by the affine hull of a facet of $P_{n}$. We denote the family of facets of $P_{n}$ by $\left\{F_{j}\right\}$, and the furthest point of the cap cut off by aff $F_{j}$ from aff $F_{j}$ by $x_{j}$. Let $d_{\partial M}\left(x_{j}, y_{j}\right)=R_{j}$ for some $y_{j} \in$ aff $F_{j} \cap \partial M$. Then (8) yields that

$$
\delta_{\mathrm{SCH}}\left(M, P_{n}\right)=\left(1+O\left(n^{-1 /(d-1)}\right)\right) \cdot \frac{\kappa_{d-1}}{2^{(d-1) / 2}(d+1)} \cdot \max _{j} \frac{R_{j}^{d+1}}{\sqrt{\kappa\left(x_{j}\right)}} .
$$

Therefore the case of Schneider's notion of distance can be handled as the case of the Hausdorff metric, only the Riemannian metric on $\partial M$ determined by $Q_{x}$ should be replaced by the Riemannian metric determined by the quadratic form $\kappa(x)^{-1 /(d+1)} Q_{x}$. 


\subsection{The Case of the Facets}

Let $\partial M$ be $C_{+}^{2}$, and assume that the coefficients of the second fundamental form are Lipschitz functions of the local parameters.

For large $n$, consider an $x \in \partial M$, and a hyperplane $H$ parallel to the tangent hyperplane at $x$ and intersecting $M$ such that distance $r$ of $H$ from $x$ is $O\left(n^{-2 /(d-1)}\right)$. Let $z \in H$, and assume that the closest point $y$ of $\partial M$ to $z$ satisfies that $d_{\partial M}(x, y)=R$ with $R=O\left(n^{-1 /(d-1)}\right)$. Then (8) yields that

$$
\pm d(y, z)=\left(1+O\left(n^{-1 /(d-1)}\right)\right) \cdot \frac{R^{2}}{2}-\left(1+O\left(n^{-1 /(d-1)}\right)\right) \cdot r
$$

and if $M=B^{d}$, then $O\left(n^{-1 /(d-1)}\right)$ can be replaced by $O\left(n^{-2 /(d-1)}\right)$ in (16). We note that the two sides of (16) are nonpositive exactly if $z \in M$.

Let $P_{(n)}$ be a polytope with at most $n$ facets minimizing $\delta_{\mathrm{H}}\left(M, P_{(n)}\right)$. We claim that

$$
\delta_{\mathrm{H}}\left(P_{(n)}, M\right)=\left(1+O\left(n^{-1 /(d-1)}\right)\right) \cdot \frac{1}{4} \cdot R_{n}(\partial M)^{2},
$$

and if $P_{(n)}$ is assumed to be inscribed or circumscribed, then $\frac{1}{4}$ should be replaced by $\frac{1}{2}$.

We may assume that $P_{(n)}$ has $n$ facets, and each facet intersects $M$. We denote the facets by $F_{1}, \ldots, F_{n}$, and let $x_{j} \in \partial M$ be the point where the outer normal is the same as for $F_{j}$, and we denote by $r_{j}$ the distance of $x_{j}$ from aff $F_{j}$ (here $r_{j}=0$ if $P_{(n)}$ is circumscribed). There exists a $y \in \partial M$ such that $d_{\partial M}\left(y, x_{j}\right) \geq R_{n}(\partial M)$ holds for $j=1, \ldots, n$, and denote by $z_{j}$ the point of aff $F_{j}$ such that $y-z_{j}$ is normal to $\partial M$. It is readily enough to consider $x_{j}$ satisfying that $d_{\partial M}\left(x_{j}, y\right)=O\left(n^{-1 /(d-1)}\right)$, and hence (16) yields that $\delta_{\mathrm{H}}\left(M, P_{(n)}\right)$ is at least the expression in (17). On the other hand, well approximating polytopes can be constructed choosing $x_{1}, \ldots, x_{n} \in \partial M$ so that for any $y \in \partial M$ there exists an $x_{j}$ with $d_{\partial M}\left(y, x_{j}\right) \leq R_{n}(\partial M)$, and letting $r_{j}=0$ if the polytope should be circumscribed, $r_{j}=\left(1+c / n^{\overline{1} /(d-1)}\right) \frac{1}{2} R_{n}(\partial M)^{2}$ with a suitable $c$ if the polytope should be inscribed, and $r_{j}=\frac{1}{4} R_{n}(\partial M)^{2}$ otherwise. Therefore Theorem 1.1 follows from (14) in case of the Hausdorff metric (and, naturally, with the improved error term for the ball).

Finally, the same alterations of the Riemannian metric on $\partial M$ are needed as for $P_{n}$ in the cases of the Banach-Mazur metric and Schneider's notion of distance.

\section{Convex $C_{+}^{2}$ Hypersurfaces}

Let $M$ be a convex body with $C^{2}$ boundary. We consider an open, Jordan measurable subset $X$ of $\partial M$ such that $\kappa(x)>0$ if $x \in \partial M$ is contained in a neighborhood of the closure of $X$.

Let $Y$ be a Jordan measurable subset of a polytope $P$, which we call a convex polytopal hypersurface. We say that $Y$ approximates $X$ if $Y$ consists of the points $y$ of $\partial P$ such that $\nu(x)$ is an exterior normal at $y$ for some $x \in X$. We say that $Y$ is inscribed if $Y \subset M$, and $Y$ is circumscribed if $Y \cap$ int $M=\emptyset$. The faces of $Y$ are the intersections of the faces of $P$ with the interior of $Y$.

Now we extend the notions of distance to $X$ and $Y$. Observe that for $x \in X$, we have

$$
h_{P}(v(x))-h_{M}(v(x))=\max _{y \in Y}\langle v(x), y-x\rangle .
$$


Hausdorff metric: $\delta_{H}(X, Y)=\max _{x \in X}\left|\max _{y \in Y}\langle\nu(x), y-x\rangle\right|$.

Banach-Mazur metric: Assume that $Y$ is inscribed, and that both int $M$ and int $P$ contain the origin. Then $\delta_{\mathrm{BM}}(X, Y)$ is the minimum of $\ln \lambda$ such that $\lambda Y$ is circumscribed. Schneider's distance: If $Y$ is inscribed into $X$, then $\delta_{\mathrm{SCH}}(X, Y)$ is the maximum volume of a cap cut off from $M$ by the affine hull of a facet of $Y$.

We justify the definitions above. Observe that if $X=\partial M$ (and hence $Y=\partial P$ ), then $\delta_{\mathrm{H}}(X, Y)=\delta_{\mathrm{H}}(M, P)$. In case of Schneider's notion of distance we have to assume that $\partial P$ is close to $\partial M$.

The only real exception is the Banach-Mazur distance, in this case the definition has been substantially altered. On the other hand, the lack of linear transformation is irrelevant in our context because linear transformations keep the family of faces.

When applying the same argument for $X$ as for $\partial M$, everything works by simply substituting $X$ for $\partial M$ except for the fact that some patches $\Omega_{i}$ intersect the boundary of $X$. Since $X$ is Jordan measurable, the proportion of the total area of these patches tends to zero, and hence the analogous asymptotic formula holds for the best approximation of $X$.

In order to obtain some estimate on the error of the asymptotic formula, we need better control of the total area of the patches near the boundary of $X$. So call a set $\sigma \subset \mathbb{R}^{d}$ rectifiable if it is the finite union of images of subsets of $\mathbb{R}^{d-2}$ by Lipschitz maps. If $\sigma$ is a rectifiable subset of $\partial M$, then for small $t$ (see pp. 42-44 of [4], but it is rather easy to prove),

$$
\left|\left(\sigma+t B^{d}\right) \cap \partial M\right|<\gamma \cdot t
$$

where $\gamma$ depends on $\sigma$. Therefore we conclude

Corollary 3.1. Let $X$ be an open, Jordan measurable subset of the boundary of $a$ convex body $M$ where $\partial M$ is $C^{2}$ and $\kappa(x)>0$ in a neighborhood of the closure of $X$. Let $Y_{n}$ be the polytopal hypersurface with at most $n$ vertices minimizing $\delta_{\mathrm{H}}\left(X, Y_{n}\right)$. Then

$$
\delta_{\mathrm{H}}\left(X, Y_{n}\right) \sim \frac{1}{4}\left(\frac{\vartheta_{d-1}}{\kappa_{d-1}}\right)^{2 /(d-1)} \cdot\left(\int_{X} \kappa(x)^{1 / 2} d x\right)^{2 /(d-1)} .
$$

If, in addition, the coefficients of the second fundamental form are Lipschitz functions of the local parameters and the boundary of $X$ is rectifiable, then

$$
\delta_{\mathrm{H}}\left(X, Y_{n}\right)=\frac{1}{4}\left(\frac{\vartheta_{d-1}}{\kappa_{d-1}}\right)^{2 /(d-1)} \cdot\left(\int_{X} \kappa(x)^{1 / 2} d x\right)^{2 /(d-1)}+O\left(\frac{1}{n^{5 / 2(d-1)}}\right) .
$$

Remark. If the $Y_{n}$ 's are assumed to be inscribed or circumscribed, then $\delta_{\mathrm{H}}\left(X, Y_{n}\right)$ is twice the value above. In addition, the same formulae hold if the number of facets is at most $n$.

If $o \in$ int $M$ and the metric is the Banach-Mazur metric, or $Y_{n} \subset M$ and the distance is Schneider's notion of distance, then the analogous formulae hold. 


\section{Lower Bound on the Error Term}

In this section we prove (2), so let $P_{n}$ be the 3-polytope with at most $n$ vertices such that $\delta_{\mathrm{H}}\left(B^{3}, P_{n}\right)$ is minimal. Suppose that, indirectly, there exist an infinite sequence $\left\{n_{k}\right\}$ and a constant $c_{1}$ such that

$$
\delta_{\mathrm{H}}\left(B^{3}, P_{n_{k}}\right)<\frac{2 \pi}{3 \sqrt{3}} \cdot \frac{1}{n_{k}}+\frac{c_{1}}{n_{k}^{2}} .
$$

Denote the regular triangle in $\mathbb{R}^{2}$ with area $2 \pi / n$ by $\tilde{T}_{n}$. Then the side of $\tilde{T}_{n}$ is $e_{n}=$ $\sqrt{8 \pi} / 3^{1 / 4} \cdot 1 / \sqrt{n}$, while the circumradius is $R_{n}=\sqrt{8 \pi} / 3^{3 / 4} \cdot 1 / \sqrt{n}$. Therefore, Lemma 2.4 yields that

$$
R_{n_{k}}\left(S^{2}\right)<\left(1+\frac{c_{2}}{n_{k}}\right) \cdot R_{n_{k}}
$$

for some constant $c_{2}$. In each formula below, the implied constant in $O(\cdot)$ depends only on $c_{2}$.

The way to find a contradiction is to construct an isometry of open subsets of $S^{2}$ and $\mathbb{R}^{2}$ with the help of (19). The construction is based on the theory of quasiconformal mappings, an idea which goes back essentially to Gauss.

Assume that $g$ is a function defined on a subset of $S^{2}$ into $\mathbb{R}^{2}$. We call $g$ a $(1+\varepsilon)$ isometry if for any two $x, y$ in the domain of definition, we have

$$
(1+\varepsilon)^{-1} d_{S^{2}}(x, y) \leq d(f(x), f(y)) \leq(1+\varepsilon) \cdot d_{S^{2}}(x, y) .
$$

Now for a spherical triangle $T$ with vertices $v_{1}, v_{2}, v_{3}$, we construct a map $g_{T, n}: T \rightarrow \widetilde{T}_{n}$ as follows: fix a linear bijective map from conv $\left\{v_{1}, v_{2}, v_{3}\right\}$ onto $\widetilde{T}_{n}$, and define $g_{T, n}$ as the composition of this linear map and the radial projection from $T$ onto $\operatorname{conv}\left\{v_{1}, v_{2}, v_{3}\right\}$. We say that $T$ is $\left(1+\varepsilon_{0}\right)$-isomorphic to $\widetilde{T}_{n_{k}}$ if $g_{T, n}$ is a $(1+\varepsilon)$-isometry.

We note that among the spherical triangles contained in a fixed spherical circle, the inscribed regular triangle, and only that, maximizes the area. We deduce

Proposition 4.1. Assume that the spherical circumradius of the spherical triangle $T$ is at most $(1+O(1 / n)) \cdot R_{n}$. Then

(i) $|T| \leq(1+O(1 / n)) \cdot\left|\widetilde{T}_{n}\right|$;

(ii) for any $\varepsilon>0$ there exists a $c(\varepsilon)>0$ such that if $n$ is large and $T$ is not $(1+\varepsilon)$-isomorphic to $\widetilde{T}_{n}$, then $|T| \leq(1-c(\varepsilon)) \cdot\left|\widetilde{T}_{n}\right|$

(iii) there exists a $0<\varepsilon_{0}<0.1$ such that if $n$ is large and $T$ is $\left(1+\varepsilon_{0}\right)$-isomorphic to $\widetilde{T}_{n}$, then each angle of $T$ is strictly between $2 \pi / 7$ and $2 \pi / 5$.

Let $\Sigma_{n}$ be the family of centers of $n$ spherical circles, with radius $R_{n}\left(S^{2}\right)$, which cover $S^{2}$. Triangulate the surface of conv $\Sigma_{n}$ using the vertices, and project radially the triangles into $S^{2}$. This way we obtain a triangulation $\Omega_{n}$ of $S^{2}$, a so-called Delone triangulation corresponding to $\Sigma_{n}$. The circumradius of each triangle in $\Omega_{n}$ is at most $R_{n}\left(S^{2}\right)$.

Proposition 4.2. For any $0<\varepsilon \leq \varepsilon_{0}$ there exists a $C(\varepsilon)$ with the following property: if $n_{k}$ is large, then all but $C(\varepsilon)$ triangles in $\Omega_{n_{k}}$ are $(1+\varepsilon)$-isomorphic to $\widetilde{T}_{n_{k}}$. 
Proof. Denote the number of triangles in $\Omega_{n_{k}}$ which are not $(1+\varepsilon)$-isomorphic to $\widetilde{T}_{n_{k}}$ by $N_{n_{k}}$. For any triangle $T$ in $\Omega_{n_{k}}$, we call the quantity $|T|-\left|\widetilde{T}_{n_{k}}\right|$ the excess of $T$. The sum of the excesses is readily zero, therefore (19) and Proposition 4.1 yield that

$$
-N_{n_{k}} \cdot c(\varepsilon) \cdot\left|\widetilde{T}_{n_{k}}\right|+n_{k} \cdot O\left(\frac{1}{n_{k}}\right) \cdot\left|\widetilde{T}_{n_{k}}\right| \geq 0
$$

In turn, we conclude the existence of $C(\varepsilon)$.

We call a vertex in $\Omega_{n_{k}}$ an $\varepsilon$-skew vertex if it is the vertex of a triangle in $\Omega_{n_{k}}$ which is not $(1+\varepsilon)$-isomorphic to $\widetilde{T}_{n_{k}}$. There exist at most $3 C(\varepsilon) \varepsilon$-skew vertices, and $\varepsilon \leq \varepsilon_{0}$ yields that each non- $\varepsilon$-skew vertex in $\Omega_{n_{k}}$ has degree six by Proposition 4.1(iii). We may assume by Proposition 4.2 and possibly taking a subsequence of $\left\{n_{k}\right\}$ that there exist $p_{1}, \ldots, p_{m} \in S^{2}, m \leq 3 C\left(\varepsilon_{0}\right)$, such that the set of $\varepsilon_{0}$-skew vertices of $\Omega_{n_{k}}$ tends to $\left\{p_{1}, \ldots, p_{m}\right\}$. Choose $r_{0}>0$ and $q \in S^{2}$ such that the spherical distance of $q$ from $\left\{p_{1}, \ldots, p_{m}\right\}$ is larger than $r_{0}$. After rotating $S^{2}$ slightly for each $\Omega_{n_{k}}$, we may assume that $q$ is a vertex of each $\Omega_{n_{k}}$, and the set of $\varepsilon_{0}$-skew vertices of $\Omega_{n_{k}}$ still tends to $\left\{p_{1}, \ldots, p_{m}\right\}$.

If $\Omega$ is a triangulation of $S^{2}$ or $\mathbb{R}^{2}$, and $u, v$ are vertices of $\Omega$, then the graph theoretic distance of $u$ and $v$ is defined as the smallest cardinality of a set $\left\{v_{1}, \ldots, v_{k}\right\}$ of vertices such that $v_{k}=v$ and each pair $\left\{\underset{\widetilde{\Omega}}{\Omega_{n}} v_{1}\right\}$ and $\left\{v_{i}, v_{i+1}\right\}, i=1, \ldots, k-1$, is formed by the endpoints of some edge. Let $\widetilde{\Omega}_{n}$ be a triangulation of $\mathbb{R}^{2}$ such that the origin $o$ is a vertex, and each triangle is congruent to $\widetilde{T}_{n}$, and let $H_{n}\left(\widetilde{H}_{n}\right)$ denote the union of the the triangles of $\Omega_{n}\left(\widetilde{\Omega}_{n}\right)$ such that the graph theoretic distance of each vertex of the triangle from $q$ (from $o$ ) is at most $r_{0} / 2 e_{n}$.

We may assume that each triangle $T$ of $\Omega_{n_{k}}$ in $H_{n_{k}}$ is $\left(1+\varepsilon_{0}\right)$-isomorphic to $\widetilde{T}_{n_{k}}$, and any vertex of $T$ has degree six in $\Omega_{n_{k}}$. We deduce that $H_{n_{k}}$ is a subset of the spherical circle of center $q$ and radius $r_{0}$, and there exists a sense-preserving bijection $h_{n_{k}}: H_{n_{k}} \rightarrow \widetilde{H}_{n_{k}}$ such that the restriction of $h_{n_{k}}$ to any triangle $T$ of $\Omega_{n_{k}}$ in $H_{n_{k}}$ is the composition of a congruence and $g_{T, n_{k}}$. In particular, $h_{n_{k}}$ is a $\left(1+\varepsilon_{0}\right)$-isometry on these triangles.

The union of the edges of $\Omega_{n_{k}}$ such that both endpoints have graph theoretic distance [ro $/ 2 e_{n_{k}}$ ] from $q$, is a simple closed curve $\gamma_{n_{k}}$. Denote a closest point of $\gamma_{n_{k}}$ to $q$ by $q_{n_{k}}$. Then the triangle inequality yields that $h_{n_{k}}$ is a $\left(1+\varepsilon_{0}\right)$-isometry of the spherical circle with center $q$ and radius $d_{S^{2}}\left(q_{n_{k}}, q\right)$, and hence

$$
d_{S^{2}}\left(q_{n_{k}}, q\right) \geq \frac{1}{1+\varepsilon_{0}} \cdot \varrho\left(\tilde{H}_{n_{k}}\right)=\frac{1}{1+\varepsilon_{0}} \cdot \frac{\sqrt{3} e_{n_{k}}}{2} \cdot\left[\frac{r_{0}}{2 e_{n_{k}}}\right]>\frac{r_{0}}{4} .
$$

Therefore each $h_{n_{k}}$ is a $\left(1+\varepsilon_{0}\right)$-isometry of the open spherical circle $B$ with center $p$ and radius $\frac{1}{4} r_{0}$.

It is time to introduce quasiconformal mappings. We need only the very basic properties, which can be found, say, in [1] or in [10]. We note that using stereographic projection from any $y \in S^{2}$ to the tangent plane at the opposite point, $S^{2}$ inherits a natural complex structure. For us, a domain is a connected open subset of $S^{2}$ or $\mathbb{R}^{2}$, and a mapping is a sense-preserving homeomorphism between open domains $S^{2}$ or $\mathbb{R}^{2}$. Assume that $\varphi$ is a Lipschitz mapping, and hence it is differentiable almost everywhere. Let $K \geq 1$. Then $\varphi$ 
is $K$-quasiconformal (see Theorem 3.5 of [10]) if for almost every $z$ where $\varphi^{\prime}(z)$ exists and for any pair $u, v$ of unit vectors in the tangent space at $z$,

$$
\left\|\varphi^{\prime}(z) u\right\| \leq K \cdot\left\|\varphi^{\prime}(z) v\right\| .
$$

We note that a $(1+\varepsilon)$-isometry is $(1+\varepsilon)^{2}$-quasiconformal, and a mapping which is $K$-quasiconformal for any $K>1$ is conformal.

We recall two basic properties of quasiconformal mappings, where the first is stated on p. 13. of [10].

Lemma 4.3. Assume that $\varphi$ is a sense-preserving homeomorphism of the domain $A$, and $\varphi$ is $K$-quasiconformal on $A \backslash \Sigma$ where $\Sigma$ is a finite set. Then $\varphi$ is $K$-quasiconformal on $A$.

We note that a sequence $\left\{\varphi_{n}\right\}$ of functions on the domain $A$ tends locally uniformly to some function $\varphi$ if $\left\{\varphi_{n}\right\}$ tends uniformly to $\varphi$ on any compact subset of $A$. Now a combination of Theorems 2.1 and 2.2 in [10] yields

Lemma 4.4. Assume that $\left\{\varphi_{n}\right\}$ is a sequence of uniformly bounded $K$-quasiconformal mappings of the domain $A \subset S^{2}$ into $\mathbb{R}^{2}$. If $d\left(\varphi_{n}(x), \varphi_{n}(y)\right) \geq \frac{1}{2} d_{S^{2}}(x, y)$ for any $x, y \in A$, then there exists a subsequence of $\left\{\varphi_{n}\right\}$ which tends locally uniformly to some $K$-quasiconformal mapping of $A$.

In particular, we may assume that $h_{n_{k}}$ tends locally uniformly to some $\left(1+\varepsilon_{0}\right)^{2}$ quasiconformal mapping $h$ of $B$ onto an open subset $\widetilde{B}$ of $\mathbb{R}^{2}$. Let $0<\varepsilon<\varepsilon_{0}$. Then there exists a finite subset $\Sigma$ of $S^{2}$ such that a suitable subsequence of the family of the at most $3 C(\varepsilon) \varepsilon$-skew vertices in $\Omega_{n_{k}}$ tends to $\Sigma$. We deduce by Lemma 4.4 that $h$ is $(1+\varepsilon)^{2}$-quasiconformal on $B \backslash \Sigma$, and hence $(1+\varepsilon)^{2}$-quasiconformal on $B$ by Lemma 4.3. This holds for any $\varepsilon>0$, therefore $h$ is actually conformal.

Now let $A$ be a closed spherical circle contained in $B$, and choose $0<\varepsilon<\varepsilon_{0}$. There exist compact subsets $\widetilde{A_{1}}, \widetilde{A}_{2}$ of $\mathbb{R}^{2}$ bounded by simple, closed Jordan curves with the properties that $\widetilde{A}_{1} \subset$ int $h(A), h(A) \subset$ int $\widetilde{A}_{2}$, and

$$
(1+\varepsilon)^{-1} \cdot|h(A)|<\left|\tilde{A}_{1}\right|<\left|\tilde{A}_{2}\right|<(1+\varepsilon) \cdot|h(A)| \text {. }
$$

The local uniform convergence yields that $h_{n_{k}}(\partial A)$ lies in $\widetilde{A}_{2} \backslash \widetilde{A}_{1}$ for large $n_{k}$, and hence

$$
(1+\varepsilon)^{-1} \cdot\left|h_{n_{k}}(A)\right|<|h(A)|<(1+\varepsilon) \cdot\left|h_{n_{k}}(A)\right| .
$$

The area of each triangle in $\Omega_{n_{k}}$ is $O\left(1 / n_{k}\right)$. In addition, all but $C(\varepsilon)$ triangles are $(1+\varepsilon)$-isomorphic to $\widetilde{T}_{n_{k}}$, and at most $c^{\prime} / \sqrt{n_{k}}$ triangles meet the boundary of $A$ where $c^{\prime}$ depends on $A$. We deduce that for large $n_{k}$,

$$
(1+\varepsilon)^{-2} \cdot|A| \leq\left|h_{n_{k}}(A)\right| \leq(1+\varepsilon)^{2} \cdot|A|,
$$

which in turn yields that $|h(A)|=|A|$ by the arbitrariness of $\varepsilon$. In particular, $h$ is conformal and preserves area, and hence an isometry between $B$ and $\widetilde{B}$. This is absurd, thus we have obtained a contradiction to (19). In other words, (2) has been finally verified. 
Remark. The corresponding properties for $r_{n}\left(S^{d}\right)$ can be verified analogously. The only essential alterations are needed in the argument for (5). If (5) does not hold for $r_{n}\left(S^{2}\right)$, then there exists a sequence of saturated packings with $n_{k}$ equal spherical circles which show that

$$
r_{n_{k}}\left(S^{2}\right)>\sqrt{\frac{2 \pi}{\sqrt{3}}} \cdot \frac{1}{\sqrt{n_{k}}}-\frac{c_{3}}{n_{k}^{3 / 2}}
$$

for some constant $c_{3}$. Here saturated means that no circle of the same radius can be added to the packing.

We note that each side of a Delone triangle $T$ is at least $2 r_{n_{k}}\left(S^{2}\right)$, while the circumradius is less than $2 r_{n_{k}}\left(S^{2}\right)$, and the area of a Delone triangle is at least the area of the regular spherical triangle $T_{n_{k}}$ with edge length $2 r_{n_{k}}\left(S^{2}\right)$. However, unfortunately, there is a triangle whose circumradius and two sides are $2 r_{n_{k}}\left(S^{2}\right)$, and whose area is $\left|T_{n_{k}}\right|$. For large $n_{k}$, the angles of this triangle are arbitrarily close to $\pi / 6$ and $2 \pi / 3$. Therefore in the analogue of Proposition 4.1, one assumes that at least two angles of $T$ are at least $2 \pi / 11$. We note that if two angles of the Delone triangle $T$ are at most $2 \pi / 11$, then the Delone triangle sharing the longest side has large area, and hence the argument can be completed as for $R_{n}\left(S^{2}\right)$.

\section{Acknowledgments}

I thank Monika Ludwig and Peter Gruber for many useful comments on polytopal approximation, and I am grateful to István Sigray for explaining the basic properties of quasiconformal mappings.

\section{References}

1. L. V. Ahlfors: Lectures on Quasiconformal Mappings. Van Nostrand, Princeton, NJ, 1966.

2. U. Betke, M. Henk, and J. M. Wills: A new approach to covering. Mathematika, 42:251-263, 1995.

3. W. Blaschke: Affine Differentialgeometrie. Springer-Verlag, Berlin, 1923.

4. K. Falconer: Fractal Geometry. Wiley, New York, 1990.

5. G. Fejes Tóth: The loosest packing of the sphere by circles. M.Sc. thesis, Roland Eötvös University, Budapest, 1970 (in Hungarian).

6. L. Fejes T6th: Lagerungen in der Ebene, auf der Kugel und im Raum, 2nd edn. Springer-Verlag, Berlin, 1972.

7. P. M. Gruber: Asymptotic estimates for best and stepwise approximation of convex bodies, I. Forum Math., 5:281-297, 1993.

8. P. M. Gruber: Aspects of approximation of convex bodies. In: Handbook of Convex Geometry A, pp. 319345. North-Holland, Amsterdam, 1993.

9. P. M. Gruber: Comparisons of best and random approximation of convex bodies by polytopes. Rend. Circ. Mat. Palermo, 50:189-216, 1997.

10. O. Lehto: Univalent Functions and Teichmüller Spaces. Springer-Verlag, Berlin, 1987.

11. K. Leichtweiß: Affine Geometry of Convex Bodies. Johann Ambrosius Barth Verlag, Heidelberg, 1998.

12. M. Ludwig: Asymptotic approximation of convex curves: the Hausdorff metric case. Arch. Math., 70:331336, 1998.

13. D. E. McClure and R. A. Vitale: Polygonal approximation of plane convex bodies. J. Math. Anal. Appl., 51:326-358, 1975 . 
14. R. Schneider: Zur optimalen Approximation konvexer Hyperflächen durch Polyeder. Math. Ann., 256: 289-301, 1979.

15. R. Schneider: Convex Bodies: the Brunn-Minkowski Theory. Cambridge University Press, Cambridge, 1993.

16. S. Tabachnikov: On the dual billiard problem. Adv. in Math., 115:221-249, 1995.

17. B. L. van der Waerden: Punkte auf der Kugel. Drei Zusatze. Math. Ann., 125:213-222, 1952.

Received April 25, 1999, and in revised form January 20, 2000, and May 31, 2000.

Online publication January 17, 2001. 